\title{
Rosetta orbiter spies possible lander site
}

Glinting pixels on comet surface raise hopes of finding hibernating Philae probe.

Elizabeth Gibney

12 June 2015

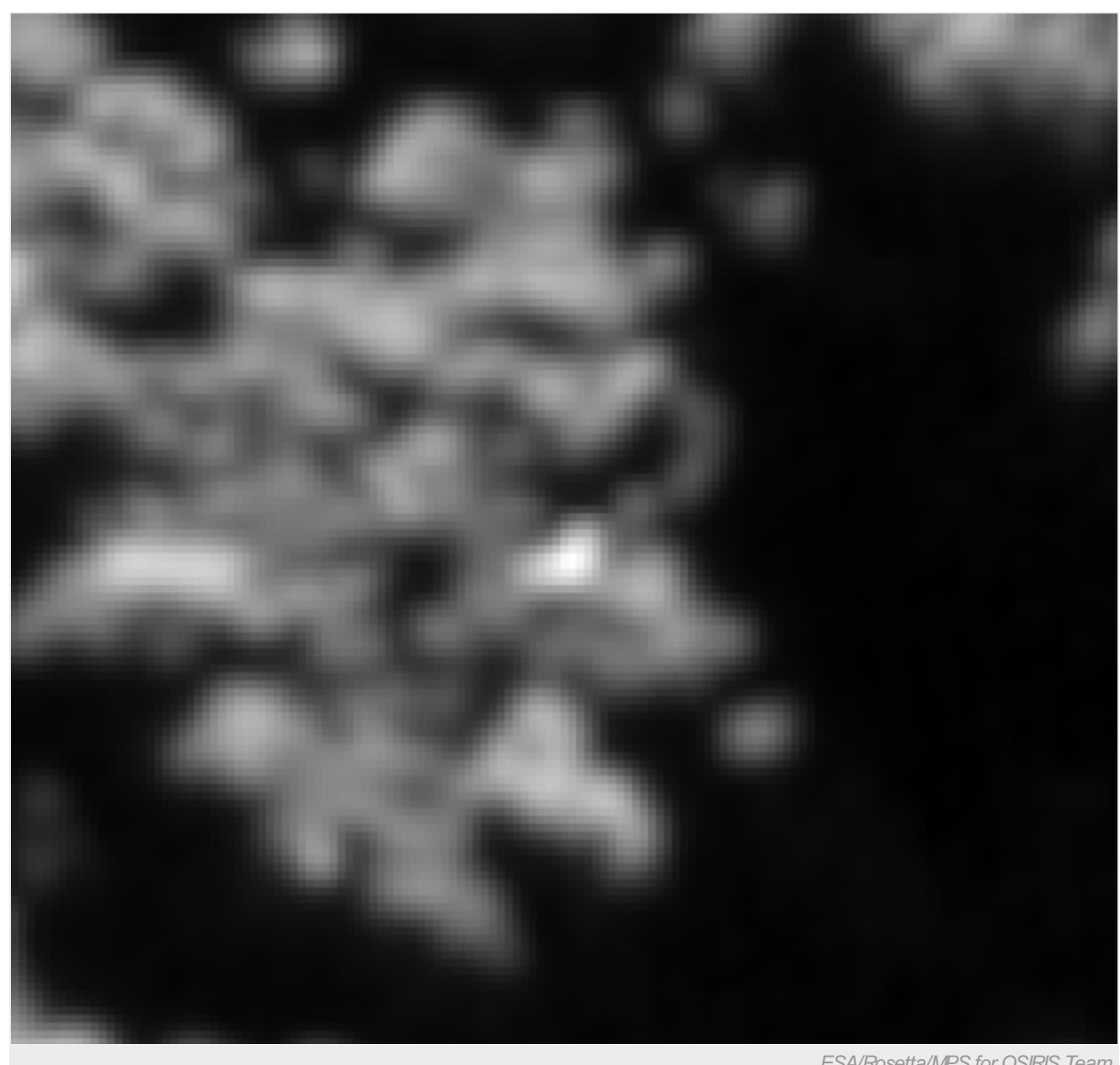

ESA/Rosetta/MPS for OSIRIS Team

Sifting through images of the surface of comet 67P/Churyumov-Gerasimenko, ESA scientists have singled out a dot of light that they say is their best guess of where Philae is.

Philae — the European Space Agency's lost comet lander — may have been spotted on the surface of the comet $67 \mathrm{P} / \mathrm{Chury}$ umovGerasimenko. 
A comparison of images of the comet surface taken by the Rosetta orbiter before and after the probe landed on 12 November last year show the appearance of a bright spot that is the best candidate yet for the sleeping craft, ESA announced this week.

Philae's landing was rough. After hitting its target, its harpoons failed and the probe bounced twice before landing in a tilted position in a shady area, and beneath a rugged-looking cliff. Without sufficient sunlight to charge its battery, the probe went into hibernation after just three days.

Since then, ESA's Rosetta and Philae teams have been working to piece together exactly where Philae's journey ended. By combining Philae's last known trajectory with data from the craft's CONSERT instrument, which sent radio waves between the lander and orbiter during Philae's short life, the scientists narrowed down the lander's probable location to a strip of roughly 16 metres by 160 metres on the comet's surface.

\section{Glints of hope}

Meanwhile, ESA scientists also have been hunting for Philae using a different approach. Researchers have looked through images taken by Rosetta's camera last December, from around 18 kilometres above the comet's surface, and have identified several possible sightings. Because of Philae's small size and shaded position, any shiny reflections from its solar panels would show up as just a few pixels across. By comparing images taken before and after the landing under the same conditions, scientists have been able to discount spots that had glinted only fleetingly. A few remaining candidates are in the vicinity of Philae's predicted home, and a favourite has now emerged.

"This bright spot is visible on two different images taken in December 2014, clearly indicating that it is a real feature on the surface of the comet, not a detector artefact or moving foreground dust speck," said Philippe Lamy, a member of the OSIRIS imager team, who worked on the images with scientists at France's Marseille Observatory (LAM) and at the Research Institute in Astrophysics and Planetology (IRAP) in Toulouse.

This white glimmer is close to, but not in, the lander's predicted resting place. However, ESA says that the strip predicted by CONSERT could yet change, as it is based on models of the comet's shape that may shift with improving data. The size and shape of the reflection does fit with predictions of Philae's probable illumination and visibility to Rosetta's radio transmitter.

\section{Other explanations}

ESA is cautious, however, not to claim that it has spotted Philae: it remains possible that the bright spot is caused by activity on the surface of the comet that has freshly exposed material, and that from the vantage point of Rosetta, Philae remains hidden by the surrounding terrain, or is simply yet to be spotted in Rosetta's reams of images.

A final answer will come only if Philae wakes up, or with a fly-by, in which Rosetta would approach closer to the comet and take images at higher resolution. However, the comet is now approaching the Sun, and is increasingly spewing gas and dust the closer it gets, so ESA says that such a fly-by may not be possible until the comet has rounded the Sun in August and the activity subsides.

Meanwhile, somewhere on the surface, Philae remains stubbornly asleep, despite now receiving what mission scientists calculate should be enough power to wake up (see 'Five factors that will decide if Philae wakes').

Knowing Philae's location would help to refine models that lander scientists are using to predict whether (or when) the craft will come back to life, and should provide valuable context to data if it does.

Even if Philae never wakes, pinpointing its location will be useful, because it would help to refine CONSERT data, which is designed to study the interior structure of the comet.

Nature I doi:10.1038/nature.2015.17752

Nature ISSN0028-0836 ESSN 1476-4687

\section{SPRINGER NATURE}

